\title{
Avances en la educación del paciente con insuficiencia cardiaca en Colombia: experiencia en el desarrollo de una herramienta educativa de la Sociedad Colombiana de Cardiología
}

\author{
Advances in the education of the patient with heart failure in Colombia: Experience \\ in the development of an educational tool of the Colombian Society of Cardiology
}

\author{
Sergio A. Gómez-Ochoa ${ }^{1 *}$, Adriana M. Jurado-Arenales ${ }^{2}$, Doris C. Quintero-Lesmes ${ }^{1}$ y Luis E. Echeverría ${ }^{2}$ \\ ${ }^{1}$ Grupo de Estudios Epidemiológicos y Salud Pública; ${ }^{2}$ Clínica de Falla Cardiaca y Trasplante Cardiaco. Fundación Cardiovascular de Colombia, \\ Floridablanca, Colombia
}

Sr. Editor,

La insuficiencia cardiaca (IC) representa en la actualidad uno de los principales retos en salud pública mundial ${ }^{1}$. A pesar de los inmensos avances logrados en su manejo desde el punto de vista farmacológico e intervencionista en las últimas décadas, la morbimortalidad en IC continúa siendo alarmantemente alta, con una incidencia de rehospitalización a 30 días de casi el $30 \%$, así como una mortalidad a cinco años hasta de un $80 \%$ en pacientes en estadio $\mathrm{D}$ de enfermedad ${ }^{2,3}$. En este contexto, destacan múltiples factores relacionados con el paciente que pueden tener un impacto negativo en sus desenlaces, especialmente la falta de adherencia a recomendaciones dietarias (dietas bajas en sodio) y fármacos ${ }^{4}$. Es por esto que recientemente se le ha comenzado a dar una mayor importancia a la educación del paciente con IC como pilar fundamental en el proceso de atención multidisciplinaria ${ }^{5,6}$. A partir de esta tendencia, nueva evidencia sobre el beneficio de estas intervenciones educativas ha comenzado a surgir en la literatura, destacando el importante rol de las intervenciones educativas lideradas por enfermeras en la incidencia de diferentes desenlaces de interés ${ }^{5}$.
Por ejemplo, la revisión sistemática de la literatura de Rice, et al. concluyó que existe evidencia que soporta un beneficio de los programas de educación en pacientes con IC, destacando una mejor calidad de vida e incluso un menor riesgo de hospitalizaciones y reingresos hospitalarios ${ }^{7}$. Sin embargo, existe una importante heterogeneidad en los diferentes abordajes educativos en esta población, por lo que se ha destacado la necesidad de evaluar intervenciones uniformes y estandarizadas con el fin de mejorar la calidad de la evidencia sobre su utilidad en la clínica ${ }^{6}$. Respondiendo a esta necesidad, desde la Fundación Cardiovascular de Colombia surgió la iniciativa de desarrollar una herramienta educativa estándar para pacientes con Falla Cardiaca en conjunto con el Capítulo de Falla Cardiaca de la Sociedad Colombiana de Cardiología.

El desarrollo de esta herramienta se llevó a cabo en dos etapas. La primera correspondió a la identificación de la evidencia y las recomendaciones más recientes acerca del diagnóstico, tratamiento y cuidados adicionales en pacientes con IC por medio de una revisión sistemática de la literatura. Posteriormente, esta evidencia fue sintetizada en un documento de manera ordenada y la información fue dividida en 14 capítulos.

\section{Correspondencia:}

*Sergio A. Gómez-Ochoa

E-mail: sagomezo.182@gmail.com
Disponible en internet: 29-11-2021 Rev Colomb Cardiol. 2021;28(5):512-514 www.rccardiologia.com 0120-5633 / @ 2021 Sociedad Colombiana de Cardiología y Cirugía Cardiovascular. Publicado por Permanyer. Este es un artículo open access bajo la licencia CC BY-NC-ND (http://creativecommons.org/licenses/by-nc-nd/4.0/).

Fecha de recepción: 21-06-2021

Fecha de aceptación: 12-08-2021

875/RCCAR.21000077 


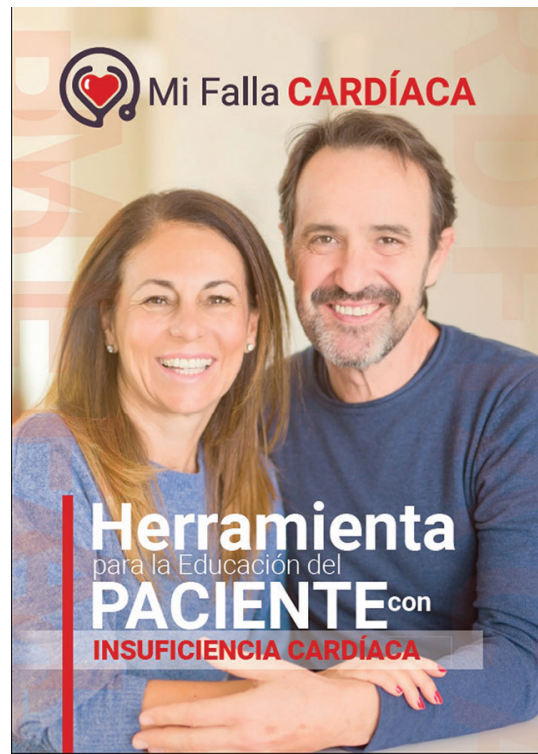

I Tabla de contenido

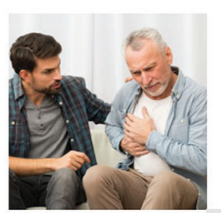

CAPÍtulo 1

Entendiendola
Insurficiencia Cardiace
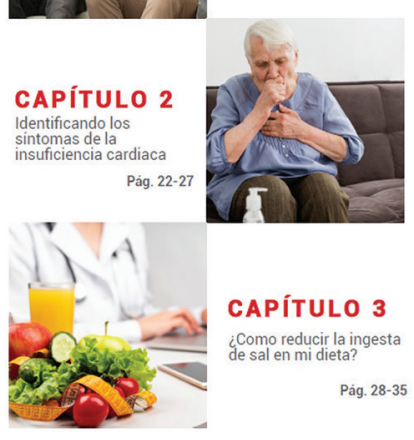

CAPÍtULO 3

Como reducir la ingesta
de sal en mi dieta?

Pág. 28-35

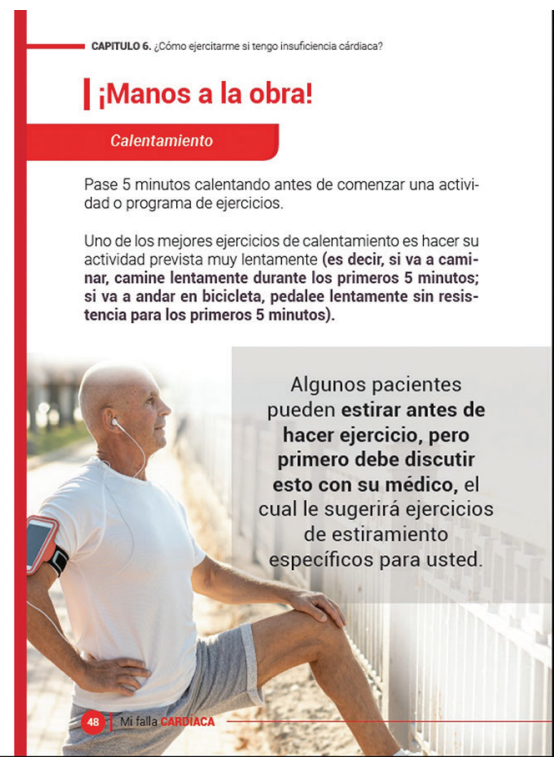

Figura 1. Muestras gráficas de la portada de la herramienta educativa, la tabla de contenido y el contenido de uno de los capítulos

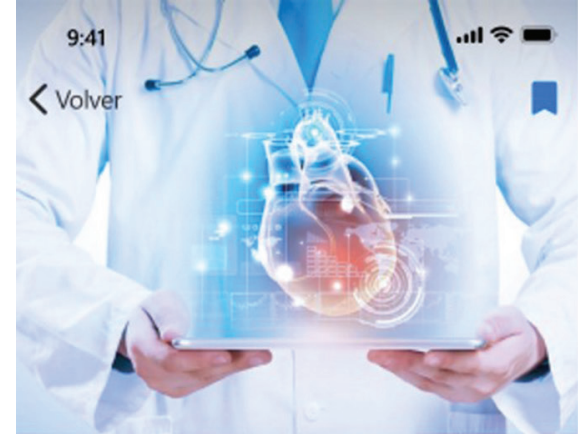

¿Qué es la Insuficiencia Cardiaca?

Namberdethutor det tento

$\therefore$ Compartir

Muchas personas creen erróneamente que la

insuficiencia cardiaca o insuficiencia cardiaca significa que el corazón ha dejado o está a punto de dejar de latir. La insuficiencia cardiaca simplemente significa que el corazón no bombea sangre a través del cuerpo como debería. A medida que la acción de bombeo de corazón se debilita, la sangre regresa a los vasos sanguíneos que rodean los pulmones y parte de esta sale a los pulmones en forma de líquido. El líquido causa congestión y dificulta la respiración. Muchas personas con insuficiencia cardiaca también tienen hinchazón de piernas y pies. Es por eso que la con insuficiencia cardiaca a veces se llama con insuficiencia cardiaca congestiva.

Ejemplos: Corazón normal vs corazón con insuficiencia

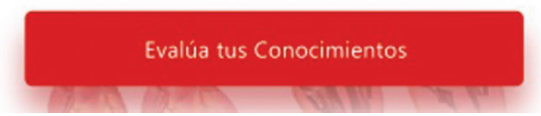

$9: 41$

$.41 \%$

Ejemplos: Corazón normal vs corazón con insuficiencia

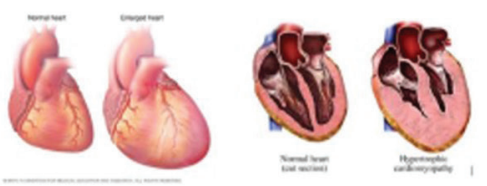

La con insuficiencia cardiaca es una enfermedad grave que puede afectar cuánto tiempo vive. Es posible que haya escuchado que algunas personas pueden morir debido a esta enfermedad. Pero con los

medicamentos adecuados en las dosis correctas y un

manejo cuidadoso, puede vivir más y sentirse mejor.

\section{¿Qué es la fracción de eyección?}

La fracción de eyección compara la cantidad de sangre en el corazón con la cantidad de sangre bombeada. La fracción o porcentaje ayuda a describir qué tan bien el corazón bombea sangre al cuerpo.
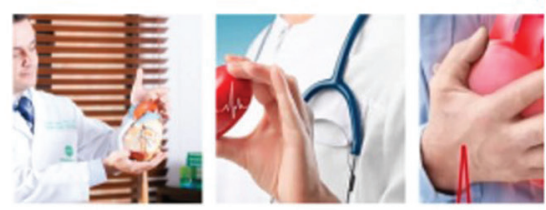

Con el tratamiento y el cuidado adecuados, una buena parte de los pacientes pueden mejorar su fracción de eyección y logran vivir una vida más longeva y

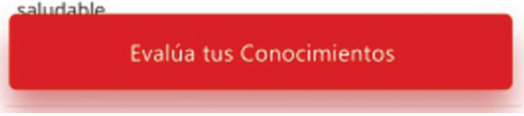

Figura 2. Muestras gráficas del capítulo 1 de la aplicación móvil basada en la herramienta educativa 
Con este material preliminar se realizó una validación de contenido con un formulario estándar, vinculando a 19 profesionales con experiencia en IC de los principales centros del país para este fin. Simultáneamente, se generó un material visual original de la mano con un diseñador gráfico, con el fin de complementar el contenido teórico del texto. Finalmente, de la mano de un ingeniero de sistemas, se generó una aplicación para teléfonos móviles con la información teórica y visual de la cartilla, además de unos módulos de evaluación de conocimientos y de valoración de contenido.

En la segunda etapa, la cartilla fue evaluada por un grupo de pacientes con IC atendidos en la Fundación Cardiovascular de Colombia (FCV). Este proceso, denominado validación facial, involucró la recopilación de comentarios y opiniones sobre la idoneidad de la herramienta desde el punto de vista visual (letra e imágenes y su distribución espacial). Para este proceso, el personal de enfermería de la FCV entrevistó individualmente a cada paciente, presentándole dos a tres capítulos de la herramienta y registrando sus percepciones haciendo uso de un formato estándar. Los cambios sugeridos por los pacientes se vieron reflejados tanto en la cartilla en físico como en la aplicación móvil.

Se obtuvo como resultado una cartilla educativa de 108 páginas (Fig. 1), en la cual se abordaron aspectos desde la definición de la IC, sus síntomas, recomendaciones alimenticias, de ejercicio físico, consumo de tabaco y alcohol, inmunizaciones, actividad sexual, consumo de medicamentos, trastornos del estado del ánimo y del sueño, viajes y recomendaciones para los cuidadores, además de un apartado para el control de citas. Por otra parte, se generó la versión de prueba de la aplicación móvil con el contenido total de la herramienta educativa (Fig. 2). Cabe destacar que tanto la herramienta educativa en físico (cartilla) como la aplicación tienen un diseño modular, lo cual facilita su edición. Esto tiene como objetivo facilitar la apropiación de la cartilla por parte de los diferentes centros de insuficiencia cardiaca del país, permitiendo la inclusión de sus logos, así como de componentes adicionales si así lo desean. Igualmente, el diseño de la aplicación definitiva ofrecerá a los diferentes centros facilidades para su personalización.

La herramienta educativa para pacientes con insuficiencia cardiaca de la Sociedad Colombiana de Cardiología representa un avance significativo con miras a la estandarización y promoción de la educación integral del paciente con IC acerca de su enfermedad.
La disponibilidad abierta de esta herramienta para todos los programas de insuficiencia cardiaca de la sociedad, así como su diseño modular y validación previa, facilitará su rápida aplicación y amplio uso. Finalmente, se espera en el futuro promover la investigación respecto al impacto de la educación impartida con esta herramienta en desenlaces clínicamente relevantes, generando evidencia de alto valor en esta área.

\section{Financiamiento}

Para la realización del presente estudio, los autores recibieron financiación por parte del gobierno Colombiano por medio del Ministerio de Ciencia, Tecnología e Innovación en el marco de la convocatoria jóvenes investigadores proyecto contrato 879-2019.

\section{Conflicto de intereses}

Los autores reportan no tener ningún conflicto de intereses.

\section{Responsabilidades éticas}

Protección de personas y animales. Los autores declaran que para esta investigación no se han realizado experimentos en seres humanos ni en animales.

Confidencialidad de los datos. Los autores declaran que en este artículo no aparecen datos de pacientes.

Derecho a la privacidad y consentimiento informado. Los autores declaran que en este artículo no aparecen datos de pacientes.

\section{Bibliografía}

1. Virani SS, Alonso A, Benjamin EJ, Bittencourt MS, Callaway CW, Carson AP, et al. Heart Disease and Stroke Statistics-2020 Update: A report from the American Heart Association. Circulation. 2020;141(9):e139-596.

2. Antohi E-L, Ambrosy AP, Collins SP, Ahmed A, Iliescu VA, Cotter G, et al. Therapeutic advances in the management of acute decompensated heart failure. Am J Ther. 2019;26(2):e222-33.

3. Chaudhry S-P, Stewart GC. Advanced heart failure: Prevalence, natural history, and prognosis. Heart Fail Clin. 2016;12(3):323-33.

4. Ruppar TM, Cooper PS, Mehr DR, Delgado JM, Dunbar-Jacob JM. Medication adherence interventions improve heart failure mortality and readmission rates: Systematic review and meta-analysis of controlled trials. J Am Heart Assoc. 2016;5(6).

5. Stamp KD, Prasun M, Lee CS, Jaarsma T, Piano MR, Albert NM. Nursing research in heart failure care: a position statement of the american association of heart failure nurses (AAHFN). Heart Lung. 2018;47(2):169-75.

6. Boyde M, Peters R. Education material for heart failure patients: what works and what does not? Curr Heart Fail Rep. 2014;11(3):314-20.

7. Rice H, Say R, Betihavas V. The effect of nurse-led education on hospitalisation, readmission, quality of life and cost in adults with heart failure. A systematic review. Patient Educ Couns. 2018;101(3):363-74. 